\title{
A photopatternable superparamagnetic nanocomposite: Material characterization and fabrication of microstructures
}

\author{
M. Suter ${ }^{\mathrm{a}, *}$, O. Ergeneman ${ }^{\mathrm{b}}$, J. Zürcher $^{\mathrm{a}}$, C. Moitzi ${ }^{\mathrm{c}}$, S. Pané ${ }^{\mathrm{b}}$, T. Rudin $^{\mathrm{d}}$, \\ S.E. Pratsinis ${ }^{\mathrm{d}}$, B.J. Nelson ${ }^{\mathrm{b}}$, C. Hierold $^{\mathrm{a}}$ \\ a Micro and Nanosystems, ETH Zürich, Tannenstrasse 3, 8092 Zürich, Switzerland \\ ${ }^{\mathrm{b}}$ Multi-Scale Robotics Lab, Institute of Robotics and Intelligent Systems, ETH Zürich, Tannenstrasse 3, 8092 Zürich, Switzerland

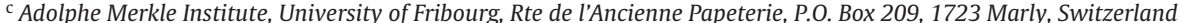 \\ d Particle Technology Laboratory, ETH Zürich, Sonneggstrasse 3, 8092 Zürich, Switzerland
}

\begin{abstract}
A superparamagnetic nanocomposite obtained by dispersing superparamagnetic magnetite nanoparticles in the epoxy SU-8 is used to fabricate microstructures by photolithography. The dispersion of the nanoparticles and the level of agglomerations are analyzed by optical microscopy, TEM (transmission electron microscope), SAXS (small-angle X-ray scattering), XDC (X-ray disc centrifuge) and XRD (X-ray diffraction). Two different phosphate-based dispersing agents are compared. In order to obtain a high-quality nanocomposite, the influence of particle concentration 1-10 vol.\% (4-32 wt.\%) on composite fabrication steps such as spin coating and UV exposure are systematically analyzed. Features with narrow widths (down to $1.3 \mu \mathrm{m}$ ) are obtained for composites with $5 \mathrm{vol}$.\% particle concentration. Mechanical, magnetic and wetting properties of the nanocomposites are characterized. These nanocomposites exhibit superparamagnetic properties with a saturation magnetization up to $27.9 \mathrm{kA} \mathrm{m}^{-1}$ for $10 \mathrm{vol} . \%$. All nanocomposites show no differences in surface polarity with respect to pure SU-8, and exhibit a moderate hydrophobic behavior (advancing dynamic contact angles approximately $81^{\circ}$ ). Microcantilevers with particle concentrations of $0-5$ vol.\% were successfully fabricated and were used to determine the dynamic Young's modulus of the composite. A slight increase of the Young's modulus with increased particle concentration from $4.1 \mathrm{GPa}$ (pure SU-8) up to $5.1 \mathrm{GPa}$ (for 5 vol.\%) was observed.
\end{abstract}

\section{Introduction}

Advances in material integration and micro and nano fabrication technologies have enabled new directions of research and development for BioMEMS and BioNEMS devices [1]. The performance of a device interacting with biological systems requires a proper selection of materials in terms of their physiochemical properties. For example, chemical stability, biocompatibility, and ease of biofunctionalization of the constituent materials are of importance.

Polymer composite materials have been highly successful in achieving different physical and chemical properties [2], as the combination of inorganic particles and polymer matrices can expand desirable material properties for new sensors, actuators and other devices [3,4]. The dispersion of superparamagnetic magnetite nanoparticles in the epoxy SU- 8 creates the possibility of manufacturing photopatternable magnetic nanocomposites (Fig. 1). Magnetic microstructures for remote actuation can be

\footnotetext{
* Corresponding author. Tel.: +41 4463255 36; fax: +41 446321462

E-mail address: marcel.suter@micro.mavt.ethz.ch (M. Suter).
}

fabricated by conventional spin coating and photolithography techniques. These microstructures are potential candidates for interfacing biological systems.

Photosensitive polymers have been modified and improved by adding an inorganic material like silica nanoparticles $[5,6]$ to change the refractive index of the material or silver nanoparticles to increase conductivity [7]. Using a photosensitive polymer in combination with magnetic particles has been reported for the fabrication of microstructures with feature widths larger than $5 \mu \mathrm{m}$ by conventional photolithography using ferromagnetic particles $[8,9]$. However, when further device miniaturization is desired the processing of composite materials that contain particles dispersed in a matrix becomes a challenge. Distribution of particles must be homogeneous within the matrix and agglomerates must be avoided in order to maintain homogenous material properties (e.g., magnetic, electrical, mechanical properties) across the microdevice and within the process batch. Characterization of particle dispersion in a composite used for microstructures is crucial. Several factors influence the agglomeration of particles: interatomic and intermolecular attractive forces favor the formation of agglomerates, and this tendency is even more prominent 

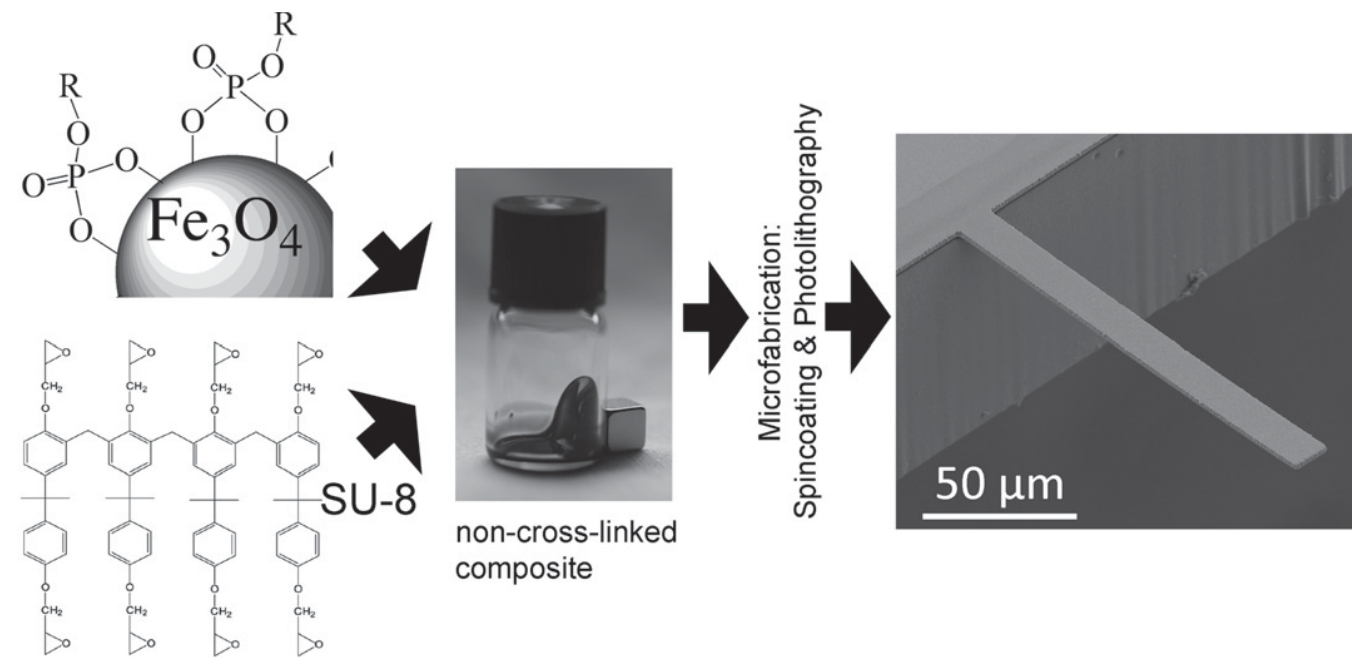

Fig. 1. Superparamagnetic magnetite nanoparticles containing a dispersing agent with a phosphate end group are mixed with the UV-sensitive epoxy SU-8 to obtain a magnetic composite. With this magnetic photocurable composite, microstructures have been fabricated using conventional microfabrication processes such as spincoating and photolithography.

when the particles exhibit magnetic properties. Magnetic particles that possess remanent magnetization strongly agglomerate due to attractive magnetic forces. This factor can be minimized by using superparamagnetic nanoparticles. Superparamagnetism is a wellknown phenomenon exhibited by ferrimagnetic and ferromagnetic single-domain nanoparticles in which no hysteresis and magnetic remanence exists. However, due to their higher surface area to volume ratio, smaller particles tend to form more agglomerates than larger ones [10]. In order to minimize particle agglomeration, different approaches have been proposed, such as functionalizing the particles' surface with emulsifiers or particles' encapsulation with polymeric or ceramic coatings [11,12]. Superparamagnetic maghemite nanoparticles have been incorporated in SU-8 with minimal aggregation using oleic acid as a surfactant [13]. However, their particle concentration is low (0.01-1 wt.\%), and for the actuation of magnetic composite microstructures a high particle concentration in the polymer is crucial to obtain sufficient forces. In our work, a dispersing agent consisting of a copolymer with a phosphate group is used to reduce agglomerations.

The polymer matrix SU-8 is an epoxy-based negative photoresist capable of being fabricated into high aspect ratio microstructures [14]. SU-8 exhibits excellent chemical stability and excellent mechanical properties [14]. SU-8 is not considered fully biocompatible according to ISO 10993, however, investigations show that toxicity derived from its degradation products is very low [15]. Furthermore, surface treatments exist that enhance cell proliferation on SU-8 [16]. Hence, this polymer is considered suitable for a large number of BioMEMS applications, such as cell culturing and biosensors $[17,18]$.

SU-8 has other favorable properties such as a high thermal stability $T_{\mathrm{g}}>200^{\circ} \mathrm{C}[19]$. The eight binding sites of the SU-8 monomer result in a highly cross-linked polymer for the fabrication of stable particle-containing microstructures. The Young's Modulus of SU-8 $(4.02 \mathrm{GPa})[19]$ is forty times smaller than that of silicon $(\sim 166 \mathrm{GPa})$ [20], resulting in a higher deflection of microstructures actuated by the same applied force, though, filler material in photocurable polymers can strongly influence fabrication parameters.

In this work, an exhaustive characterization of the composite material and its processability is carried out providing relevant information for the further development of this kind of material. Since agglomeration is one of the most significant issues to be addressed in the fabrication of nanocomposites, a focus on particle agglomeration as a function of particle concentration from 1 to
$10 \mathrm{vol} . \%$ (4-32 wt.\%) is investigated. Moreover, the influence of two different dispersing agents on the nanoparticles agglomerations is also shown. Despite the existence of some works dealing with the fabrication of composites of photopatternable matrices containing nanoparticles [21], there is scarce literature regarding the dispersion of nanoparticles inside such matrices [22]. Relevant physical properties of the composite such as magnetism and mechanical stiffness are also characterized as a function of particle concentration since such properties play an important role for the use of the material in its actuation. Additionally, the composites' feature resolution and its surface polarity is analyzed since the wettability of the surface will determine the further material functionalization. While the details of the microstructure fabrication process and the remote magnetic actuation of the composite cantilevers have been previously investigated [23], here, we investigate further the influence of particle concentration on the fabrication process. Due to UV-absorption of the particles in the composite, the exposure dose of the composite must be adjusted for each particle concentration. Furthermore, a higher particle concentration increases the viscosity of the initial non-cross-linked composite and must be considered for the spin-coating process.

\section{Experimental}

\subsection{Nanoparticle dispersion (magnetic suspension)}

The nanoparticle dispersion is developed in cooperation with Chemicell GmbH (Berlin, Germany). A wet-chemical synthesis from iron-II/III-salt dissolution is used to fabricate the magnetite particles. The particles are washed with $\mathrm{H}_{2} \mathrm{O}$, coated with the dispersion agent and transferred into the organic solvent $\gamma$-butyrolacton(GBL) to form a stable dispersion. GBL is used, because it is a solvent for SU-8. A stable dispersion (even after 10 months) of up to $280 \mathrm{mg} \mathrm{ml}^{-1}$ is obtained. Such a high concentration in the dispersion is crucial for the fabrication of composites with particle concentrations up to $10 \mathrm{vol} . \%$.

\subsection{Composite and microstructure fabrication}

After 10 min sonication in a conventional ultrasonic-bath, the nanoparticle dispersion is mixed (ratio 2:3) with the dissolved SU-8 (with a low solvent content, 48\% GBL) from MicroChem Corp. (Newton, USA). Additionally, the composite is mixed for $10 \mathrm{~min}$ in 
a planetary mixer (dual asymmetric centrifugal mixer, DAC 150 FVZ, Hausschild) with an initial 2 min speed ramp (0-3000 rpm). The composite is treated by ultrasonic sound (Vibracell VCX 600 Sonics \& Materials Inc.) for $20 \mathrm{~min}$ and then spin-coated onto a support glass wafer. The microfabrication process has been previously detailed [23].

\subsection{TEM images}

The particle diameter and the agglomerate diameter are determined from several TEM images (Philips CM12 with a tungsten filament at $100 \mathrm{kV}$ ) using the software Image $1.42 \mathrm{q}$. Grinding and dimple-grinding (Gatan 656) are used to prepare thin film slices of the composite. With the precision ion polishing system (PIPS) Gatan 691 a hole is etched in the sample by two focused argon ion beams. At the edge of the hole the probe is thin enough for TEM observation.

\subsection{SAXS measurements}

The SAXS equipment consists of a S-MAX3000 instrument from Rigaku Innovative Technologies (Auburn Hills, MI, USA). The radiation is produced by a high-intensity micro-focus sealed tube X-ray generator (Rigaku MicroMax-002+) with a copper anode (wavelength $0.154 \mathrm{~nm}$ ). A pair of multilayer reflectors is used to produce a monochromatic beam which is collimated by a three pinhole system. In the fully evacuated camera the sample is placed at a distance of $1525 \mathrm{~mm}$ from the detector. The diameter of the incident X-ray beam at the sample position is approximately $0.8 \mathrm{~mm}$. The sample absorption is measured by a photodiode which is mounted on the beam-stop. The scattered intensity is detected by a fully integrated two-dimensional multi-wire proportional counter (Rigaku Triton) which makes highly sensitive measurements from isotropic and anisotropic materials possible. The diameter of the sensitive area of the detector is $200 \mathrm{~mm}$. The range of accessible scattering vectors $q$ which is defined as

$q=\left(\frac{4 \pi}{\lambda}\right) \sin \left(\frac{\theta}{2}\right)$

with $\lambda$ being the $X$-ray wavelength and $\theta$ being the scattering angle, is $0.07<q<4.5 \mathrm{~nm}^{-1}$. For the model fitting approach to calculate the bi-lognormal distribution of homogeneous spheres program SASfit (by Joachim Kohlbrecher and Ingo Bressler, Paul Scherrer Institute, $\mathrm{CH})$ was used.

\subsubsection{XRD measurements}

XRD measurements are taken from particles, which were dried from the initial particle dispersion in a vacuum oven under $\mathrm{N}_{2}$ $\left(100^{\circ} \mathrm{C}\right.$ at $\left.20 \mathrm{mbar}\right)$ to avoid changes in the structure of the particles. XRD patterns are recorded from three probes with a Bruker D8 Advance diffractometer $(40 \mathrm{kV}, 40 \mathrm{~mA}, \mathrm{CuK} \alpha)$ at $2 \theta=10-80^{\circ}$. By the fundamental parameter approach with the Rietveld refinement [24], crystallite sizes of $\mathrm{Fe}_{3} \mathrm{O}_{4}$ (ICSD 028664) are obtained using TOPAS 3.0 (Bruker).

\subsection{XDC measurements}

The hydrodynamic diameter of the initial particle dispersion is measured with a Brookhaven Instrument X-ray disc centrifuge (3000 rpm, $300 \mathrm{~min}, 22^{\circ} \mathrm{C}$ ). $1.2 \mathrm{ml}$ particle dispersion is diluted with $23.8 \mathrm{ml} \mathrm{GBL}$ and sonicated in a conventional ultrasonic-bath for $10 \mathrm{~min}$. To ensure solvent compatibility a polycarbonathomolite $\mathrm{H}-911$ disc is used for the XDC measurements.

\subsection{Magnetic measurements}

The magnetic characteristics of the nanoparticles and composite structures are obtained by measuring their $\mathrm{M}-\mathrm{H}$ loops. The magnetic characterization of the nanoparticles was done using a vibrating sample magnetometer (VSM) (Micromag 3900, Princeton Measurement Corporation). The nanoparticles are weighed using a microbalance prior to the measurement. The composite structures are measured using an alternating gradient magnetometer (AGM) (Micromag 2900, Princeton Measurement Corporation) due to its higher sensitivity. Films of composite are prepared with known dimensions for the magnetic measurements.

\subsubsection{Viscosity and layer thickness measurements}

A prototype of a rod-type sensor for viscosity measurement AST100 from Brookfield (Middleboro, MA, USA), developed at Institute of Mechanical Systems, ETH Zurich is used. Due to the low sample volume the accuracy of the measurements are $\pm 20 \%$. The layer thicknesses of the different spin-coated and patterned composites are determined from 4 to 7 measurements, using a profilometer Tencor P10 from KLA-Tencor Corporation (San Jose, USA).

\subsection{UV-transmittance measurement and surface investigation}

The spectra of the transmittance of composite layers with different particle concentrations (1, 2, 3, 5 and 10 vol.\%) have been measured. The measured thicknesses of the samples are shown in Table 2. To analyze the light transmittance with a UV/VIS spectrometer (Cary 500, Varian) exposed composite areas have been released from the substrate and placed on fused silica glass supports. Fused silica has a transmittance of $90 \%$ above $200 \mathrm{~nm}$ and, therefore, well suitable as a substrate for transmittance measurements. The wavelength dependant absorption of the fused silica substrate was eliminated by a background measurement. The top and bottom surfaces of microcantilevers with 5 and $10 \mathrm{vol} . \%$ are observed by secondary electron microscope (SEM) (FEI Quanta 200 FEG).

\subsection{Pattern transfer in the composite}

The widths of the fabricated structures and mask patterns have been measured on three different probes using a Leica DM4000 optical microscope calibrated with a $70 \mu \mathrm{m}$ circular standard sample.

\subsection{Contact angle measurements}

Dynamic contact angle measurements were performed on a Krüss DSA 100 (Krüss, Germany). For dynamic contact angle (advancing $\left(\theta_{\mathrm{a}}\right)$ and receding $\left(\theta_{\mathrm{r}}\right)$ ) measurements the drop volume was increased and decreased with a speed of $15 \mu \mathrm{Lmin}^{-1}$. This leads to low-rate contact angle measurements with advancing contact-line speeds below $0.012 \mathrm{~mm} \mathrm{~s}^{-1}$. Receding contact-line speeds are slightly higher on strongly pinning surfaces at around $0.03 \mathrm{~mm} \mathrm{~s}^{-1}$. For the advancing drop, one movie with 100 frames and, for the receding drop, one movie with 250 frames was recorded.

\subsubsection{Dynamic Young's modulus}

The cantilevers are actuated by thermal noise under vacuum pressure $\left(4.6-22 \times 10^{-3} \mathrm{~Pa}\right)$. The vibrations are measured by a Laser-Doppler-Vibrometer (Polytec GmbH, MSA-500). The signal is transformed into the frequency domain by the means of Fast Fourier Transformation. In order to minimize noise, the frequency spectra are averaged over 300 measurements. White noise and $1 / f$ noise are eliminated and the square amplitude frequency spectra 


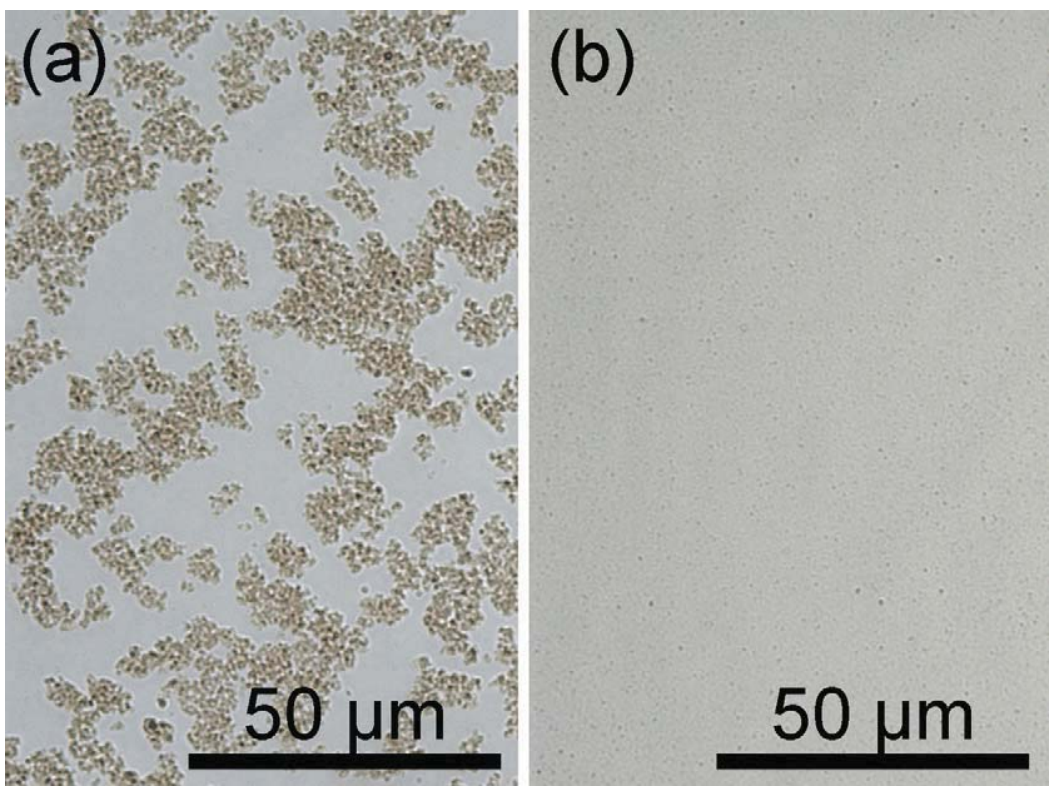

Fig. 2. Light microscope images in transmission mode of a polymerized composite containing 2 vol.\% ( 8 wt.\%) $\mathrm{Fe}_{3} \mathrm{O}_{4}$ particles with a film thickness of $1.75 \pm 0.15 \mu \mathrm{m}$. (a) Magnetite particles are coated by diphosphate and form agglomerates (mean diameter $>5 \mu \mathrm{m}$ ) in the polymer. (b) If the magnetic particles are coated with a dispersant agent containing a copolymer with a phosphate group, agglomerates are no long visible under light microscopy.

are fitted by a Lorentzian curve shape. The length $L$ of the cantilevers is measured with an optical light microscope Leica DM4000. The thickness $h$ is determined by a profilometer Tencore P10. The density of the composite is assumed to be a perfect dispersion of the incorporated nanoparticles in the SU-8:

$\rho=\chi \rho_{\mathrm{Fe}_{3} \mathrm{O}_{4}}+(1-\chi) \rho_{\mathrm{SU}-8}$

where $\rho_{\mathrm{Fe}_{3} \mathrm{O}_{4}}$ is the density of $\mathrm{Fe}_{3} \mathrm{O}_{4}$ nanoparticles $\left(5180 \mathrm{~kg} \mathrm{~m}^{-3}\right)$, $\rho_{\mathrm{SU}-8}$ the density of SU- $8\left(1190 \mathrm{~kg} \mathrm{~m}^{-3}\right)$, and $\chi$ is the volume fraction of the nanoparticles in the composite.

\section{Results and discussion}

\subsection{Dispersion}

To achieve homogenous mechanical and magnetic properties in the composite microstructures, the nanoparticles must be well dispersed within the host matrix. Hence, the use of a proper dispersing agent is critical. Despite the high number of possibilities (surfactants, polymers, complexing agents), the proper dispersant must fulfill important requirements such as a strong attachment to the nanoparticle surface and an optimal chemical affinity of the surfactant with the surrounding matrix. The importance of the particle functionalization is illustrated in Fig. 2. In this work, two different phosphate-containing dispersing agents are used. Phosphate groups are known to adsorb strongly onto magnetite surfaces [25]. However, the presence of such functional groups does not guarantee dispersion stability. Using diphosphate as a dispersant, relatively large magnetite agglomerates greater than $5 \mu \mathrm{m}$ in size can be observed under an optical microscope after mixing (Fig. 2(a)). Such a composite would result in microstructures with rough surfaces and non-uniform mechanical properties. When magnetite nanoparticles are functionalized with a dispersant consisting of a linear copolymer containing a phosphate group, agglomerates are no longer observed (Fig. 2(b)) by optical microscopy.

Further investigation of the composite dispersion with TEM images was performed. Fig. 3 shows a TEM image of the crosssection of a composite layer containing 3 vol.\% (12 wt.\%) and 5 vol.\% (18 wt.\%) magnetite particles. In Fig. 4, a close up view of the com- posite is depicted. A clear dispersion of $\mathrm{Fe}_{3} \mathrm{O}_{4}$ particles in the $\mathrm{SU}-8$ matrix with a low level of agglomeration can be observed. The agglomerate diameter distribution of a composite with 5 vol.\% particle concentration is measured from TEM images ( 366 counts) and shown in Fig. 5(a). The agglomerate's average count diameter is $43.5 \pm 20.4 \mathrm{~nm}$.

The particle diameter distribution measured from TEM images is depicted in Fig. 5(b). The particles' average count diameter is $11.4 \pm 3.4 \mathrm{~nm}$ (800 particles measured). From a two-dimensional TEM image, it is difficult to estimate the agglomerate sizes because the particles and agglomerates can overlap within the $\sim 100 \mathrm{~nm}$ thick slice.

In order to quantitatively determine the size distribution of particles and agglomerates, small angle X-ray scattering (SAXS) measurements were performed. Composite films with a typical thickness between $1.54 \mu \mathrm{m}$ and $2.23 \mu \mathrm{m}$ were measured using a transmission geometry with a measurement time of $1 \mathrm{~h}$. The scattered intensities were azimuthally averaged and corrected for the sample absorption (the raw intensities were divided by the sample transmission). Small differences in sample geometry were taken into account by dividing the scattering curve with the thickness of the sample at the position of the X-ray beam. Background scattering corresponding to the polymer matrix was taken into account by subtracting the scattering of a sample that did not contain particles. Finally, the scattering curves were normalized by the $\mathrm{Fe}_{3} \mathrm{O}_{4}$ concentration, since particle count density must be considered. Fig. 6 shows characteristic curves that provide information on the agglomeration state of the particles for different particle concentrations in the composite. The SAXS curves show two distinct slopes indicating the presence of two particle/agglomerates sizes. The SAXS curves for samples, containing 1-10 vol.\% of $\mathrm{Fe}_{3} \mathrm{O}_{4}$, overlap, indicating that the agglomeration states of particles in the composite are independent of the particle concentration. Because of the higher scattered intensity, the noise of the SAXS curve is lower for increasing particle concentration. At the same time the scattering of the X-ray at very small angles is significantly suppressed at $\mathrm{Fe}_{3} \mathrm{O}_{4}$ concentrations of 5 and $10 \mathrm{wt} . \%$. This is due to particle interactions which cannot be neglected at these concentrations. Small angle X-ray scattering does not provide information on the nature of the interaction but can distinguish between repul- 


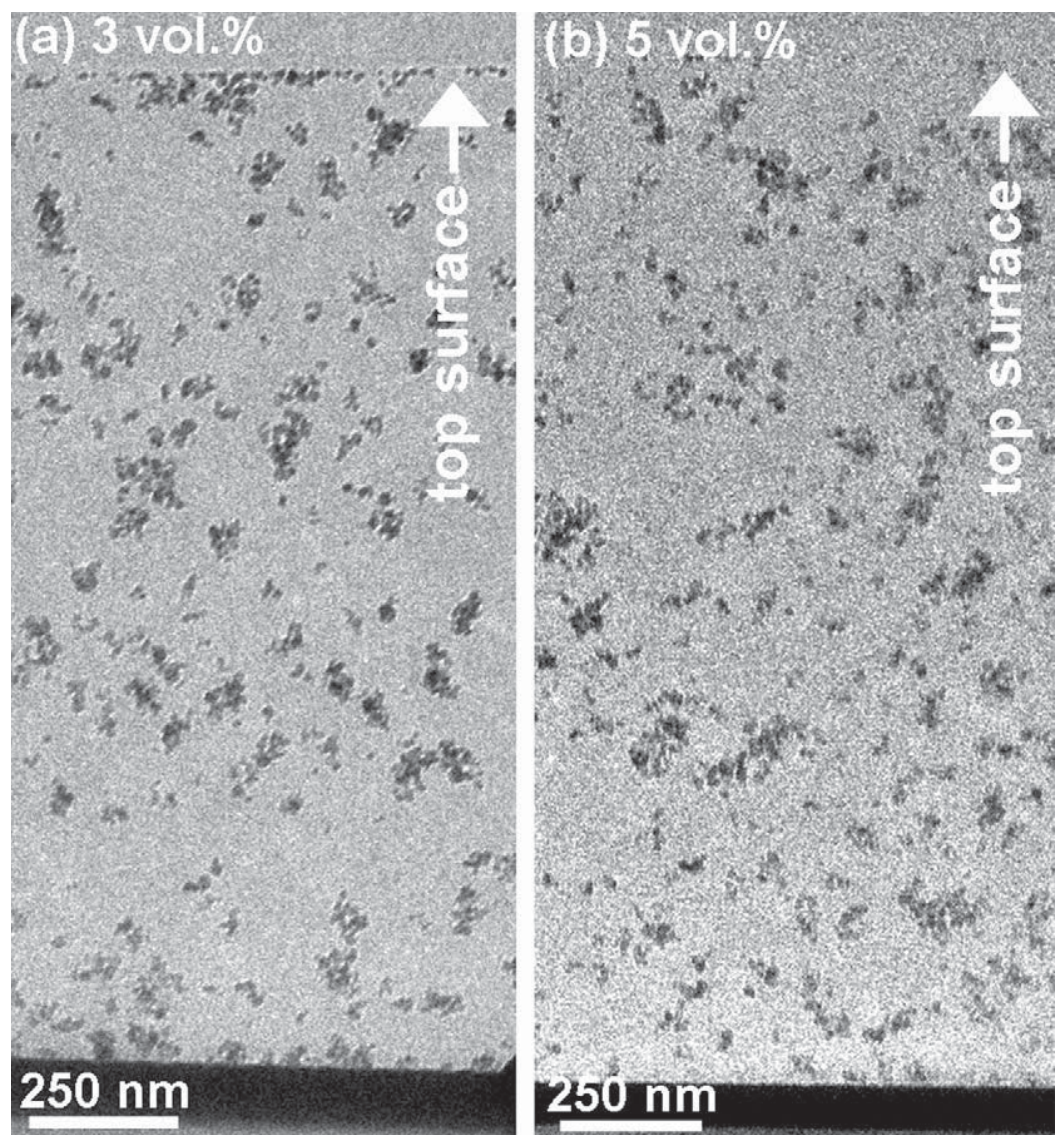

Fig. 3. TEM image of a cross-section of the polymer composite film with (a) 3 vol.\% (12 wt.\%) and (b) 5 vol.\% (18 wt.\%) $\mathrm{Fe}_{3} \mathrm{O}_{4}$ particle concentration (sample thickness $\approx 100$ nm).

sive and attractive interaction potentials. In general, the scattering intensity at very small angles increases if the particles attract each other, while it is suppressed if they repel each other. The simplest type of interaction, which is often a good approximation for stable particle dispersions, is the so-called hard sphere potential. Here particles are not allowed to overlap but do not interact when they are separated. For such a system the scattering at very small angles is suppressed by $32 \%$ at a volume fraction of particles of $5 \%$ and by $54 \%$ at a volume fraction of $10 \%$ [26]. Thus, one has to expect that particle interactions have a significant effect on the scattering curve at high particle concentration. It must be noted that particle agglomeration would have the opposite effect, which would mean an increase of the scattered intensity at small scattering angles. The SAXS data clearly shows that the particles interact via an effectively repulsive interaction potential. Even at the highest particle fill considered here, the nanoparticles are still well dispersed.

For a further analysis of the SAXS data, effects of particle interactions must be excluded. Therefore, the sample containing 3 vol.\% (12 wt.\%) of $\mathrm{Fe}_{3} \mathrm{O}_{4}$ was chosen as a compromise between enhanced data statistics and small particle interactions. Two different approaches to calculate the size distribution of the scattering objects (i.e. single particles or agglomerates) were used. First, an Indirect Fourier Transformation (IFT) $[27,28]$ was applied to calculate the size distribution by assuming homogeneous spherical particles. Second, a model fitting approach was used to calculate the bi-lognormal distribution of homogeneous spheres that gives the best fit to the experimental data. In Fig. 7, both volume weighted distributions are shown. Within the experimental error of the method, both results overlap. The main fraction of particles is between 10 and $20 \mathrm{~nm}$ in diameter. However, there is a significant content of particles or agglomerates which are larger. The distribution of diameters extends up to $80 \mathrm{~nm}$. The overlap of the scattering

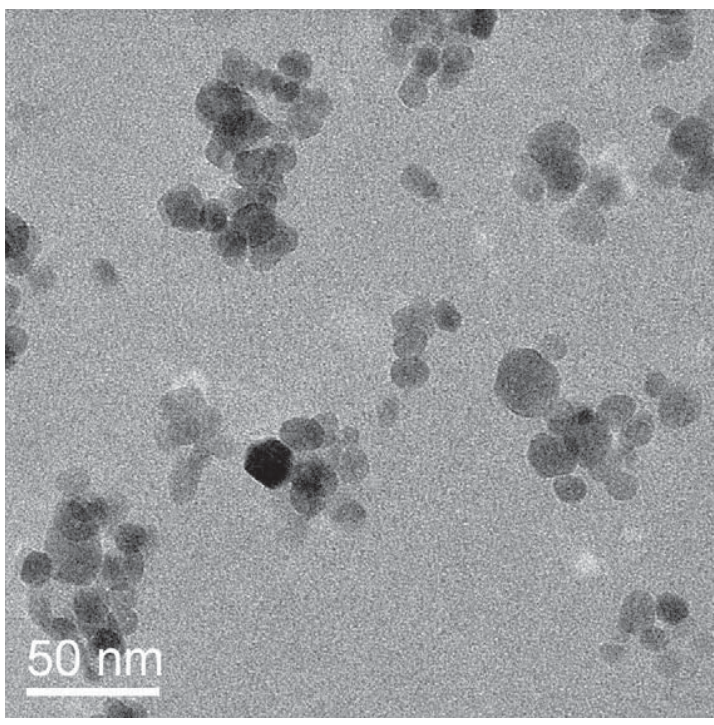

Fig. 4. TEM image of the particles/agglomerates in the composite with 5 vol.\% $\mathrm{Fe}_{3} \mathrm{O}_{4}$ nanoparticles.

curves measured with different particle fillings shows that the same size distribution is present at all $\mathrm{Fe}_{3} \mathrm{O}_{4}$ concentrations being investigated. The distribution originates either from the size distribution of individual particles or from agglomerates that cannot be broken up by the homogenization procedure and which are present at all particle fillings. Compared with the size distribution of the particles measured by TEM, the first peak fits very well to the individual particles size distribution, whereas the second hump at $40-50 \mathrm{~nm}$ corresponds to existing agglomerates. 

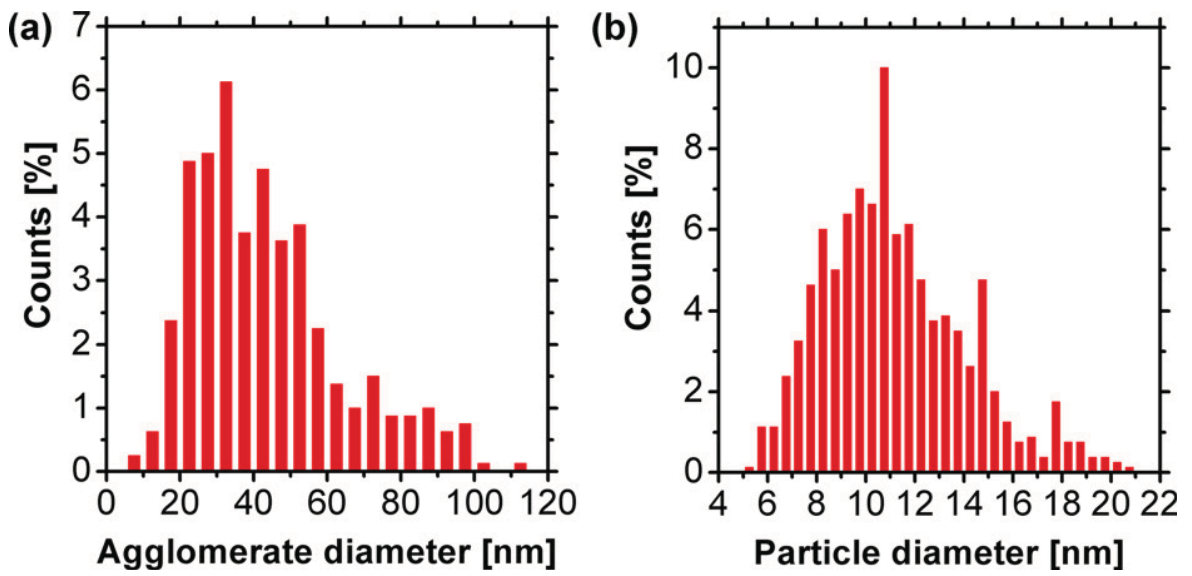

Fig. 5. (a) Agglomerate diameter distribution from TEM images (366 counts) of a composite with 5 vol.\% particle concentration. The average diameter is $43.5 \pm 20.4 \mathrm{~nm}$. (b) Particle diameter distribution measured from TEM images (count distribution). The particles have a mean count diameter of $11.4 \pm 3.4 \mathrm{~nm}$ ( 800 counts).

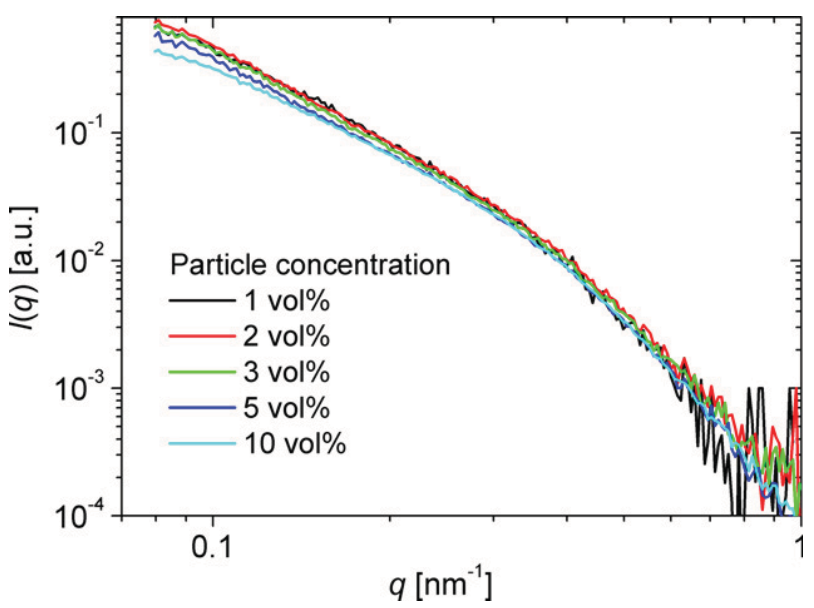

Fig. 6. SAXS measurements: 0 vol.\% probe is subtracted, the thickness of the sample was considered and the data are normalized with respect to particle concentration. The overlap of the curves confirms the expected particle concentrations. The similar shape of the curves shows that the agglomeration states of particles in the composite are independent of the concentration.

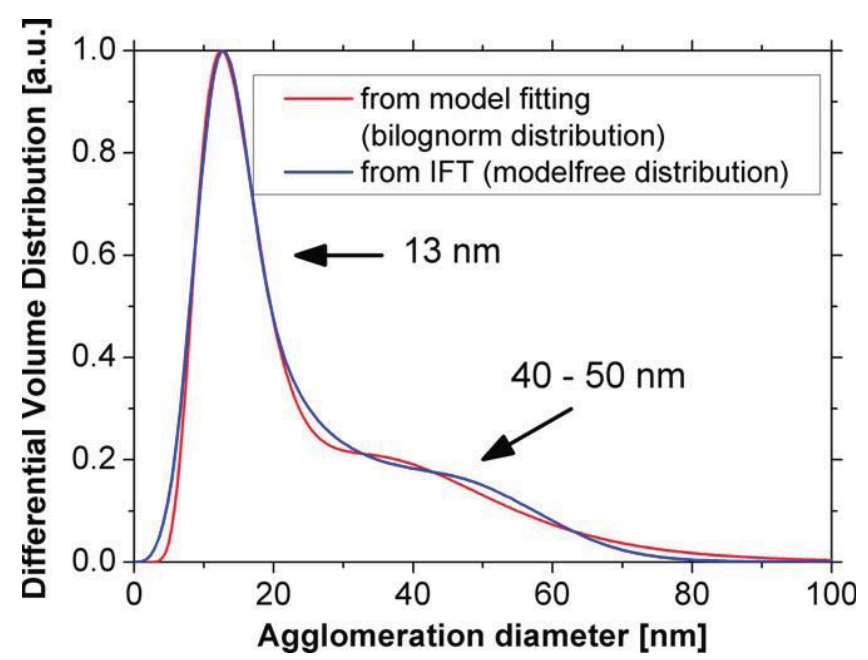

Fig. 7. Differential volume distribution of particles and agglomerates in composite modeled from SAXS measurements. Measurement range: 1-100 nm.

These agglomerates can also be present in the initial nanoparticle dispersion (magnetite particles dispersed in the solvent $\gamma$-butyrolacton (GBL). The measurement of hydrodynamic parti-

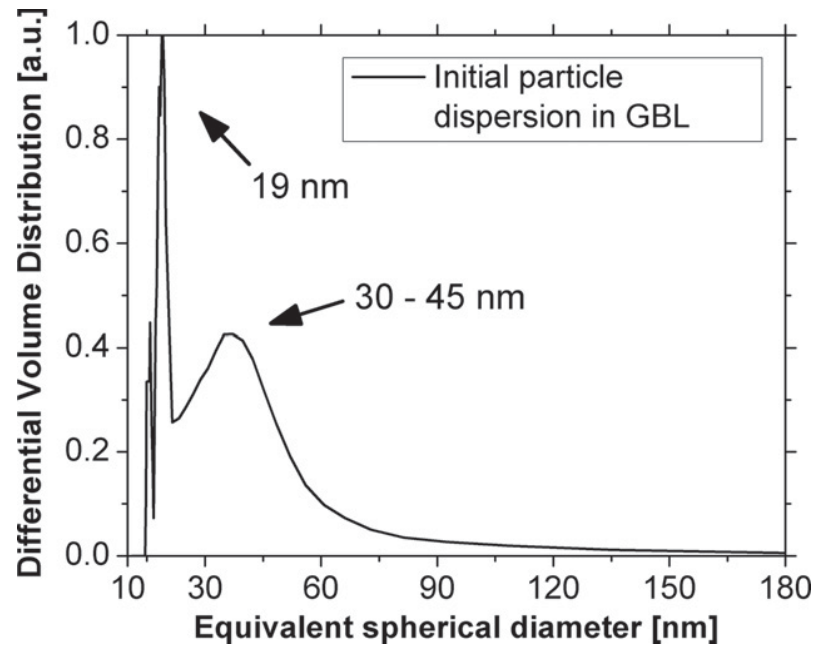

Fig. 8. Differential volume distribution of particles and agglomerates in initial particle dispersion (solvent: GBL) measured with XDC (surfactant layer contributes to the sizes). Measurement range: $>10 \mathrm{~nm}$.

cle/agglomerate sizes from the X-ray disc centrifuge (XDC) depicted in Fig. 8 shows the diameter distribution of the particles in the initial nanoparticle dispersion before mixing with the photosensitive polymer SU-8. XDC measures hydrodynamic diameters of agglomerates including the surfactant layer and the fluid boundary layer. Therefore, the sizes are expected to be larger compared to TEM and SAXS. The measurement shows two peaks: one at $19 \mathrm{~nm}$, which corresponds to the single particle diameter or agglomerates with low primary particle number, and a second peak around $38 \mathrm{~nm}$ (30-45 nm), which matches with the agglomerate size measured from SAXS. This indicates that in the initial magnetic suspension agglomerates are present, which cannot be further broken by the used mixing methods.

Nanoparticles have been analyzed by XRD measurements as shown in Fig. 9. The XRD-spectra fits to magnetite $\mathrm{Fe}_{3} \mathrm{O}_{4}$ (ICSD 028664). The main diffraction peaks are indicated in the graph. The spectra of magnetite and maghemite $\gamma-\mathrm{Fe}_{2} \mathrm{O}_{3}$ (ICSD 87119) are very similar. Despite the lack of the additional peaks of maghemite (210) at $23.7^{\circ}$ and $(211)$ at $26.1^{\circ}$ the presence of maghemite cannot be completely excluded. Other iron oxide phases such as wustite FeO (ICSD 82233) and hematite (ICSD 066756) can be excluded. The average crystallite size of $\mathrm{Fe}_{3} \mathrm{O}_{4}$ (ICSD 028664) measured from the main peak $(311)\left(34.6-36.6^{\circ}\right)$ is $13.1 \pm 0.5 \mathrm{~nm}$. 


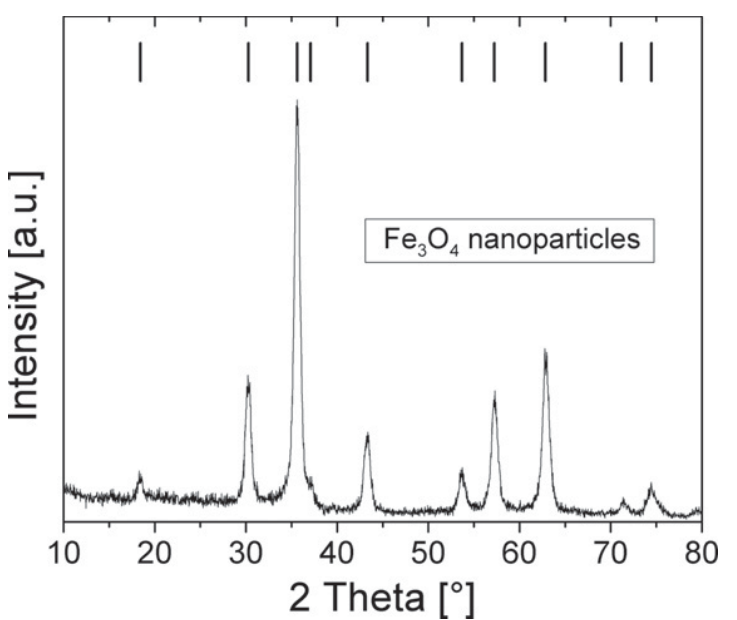

Fig. 9. XRD pattern of the magnetite $\left(\mathrm{Fe}_{3} \mathrm{O}_{4}\right)$ nanoparticles. The magnetite peak positions (ICSD 028664) are indicated.

Table 1

Comparison of particle and agglomerate diameters measured by different measurement methods.

\begin{tabular}{lll}
\hline Measurement method & $\begin{array}{l}\text { Particle } \\
\text { diameter }[\mathrm{nm}]\end{array}$ & $\begin{array}{l}\text { Agglomerate } \\
\text { diameter }[\mathrm{nm}]\end{array}$ \\
\hline $\mathrm{XRD}_{\text {crystal size }}$ & 13.1 & - \\
$\mathrm{TEM}_{\text {volume average }}$ & $12.4^{\mathrm{a}}$ & $52^{\mathrm{b}}$ \\
$\mathrm{SAXS}^{\mathrm{c}}$ & 13 & $40-50$ \\
$\mathrm{XDC}_{\text {hydrodynamic diameter }}{ }^{\mathrm{d}}$ & 19 & $30-45$ \\
\hline
\end{tabular}

a Taken from 800 measurements.

b Taken from 366 measurements.

c Main peaks from Fig. 7.

d Main peaks from Fig. 8.

Particle and agglomerate size measurements by SAXS, XRD and XDC provide information on the diameter in terms of a volume or mass distribution, whereas TEM measurements result in a count size distribution. For comparison the count average diameter of the TEM measured particles $(11.4 \pm 3.4 \mathrm{~nm})$ must be calculated into the volume average diameter resulting in $12.4 \mathrm{~nm}$. The volume average diameter of the agglomerates is $52 \mathrm{~nm}$. A comparison of measured diameters from SAXS, XRD and TEM methods has been previously described in detail [29]. The different particle and agglomerate sizes are summarized in Table 1 . The crystal size measured by XRD is in good agreement with the particle diameter measured in the composite by TEM and SAXS. Considering the hydrodynamic layer, as lightly larger diameter from the XDC measurements is expected and corresponds as well to the particle diameters determined by the other methods. The volume average agglomerate sizes measured by TEM fit to the main agglomerate sizes of the SAXS measurements. The comparison between SAXS measurements of the composite and the XDC measurements from the initial particle dispersion shows that some agglomerates are already present in the initial dispersion. The main agglomerate size during composite mixing does not increase significantly.

\subsection{Magnetic properties}

Fig. 10 shows the $\mathrm{M}-\mathrm{H}$ curve for $\mathrm{Fe}_{3} \mathrm{O}_{4}$ nanoparticles. The particles show negligible hysteresis, as expected, indicating superparamagnetic behavior at room temperature. At the maximum applied field $\left(800 \mathrm{kA} \mathrm{m}^{-1}\right)$ the nanoparticles reach saturation and magnetization was measured as $277 \mathrm{kA} \mathrm{m}^{-1}\left(53.5 \mathrm{emug}^{-1}\right)$ and is similar to previously reported value of the same probe [23]. Saturation magnetization of $\mathrm{Fe}_{3} \mathrm{O}_{4}$ nanoparticles with different fabrication process are in the same range $\left(60.1 \mathrm{emu} \mathrm{g}^{-1}\right.$ for $11.5 \mathrm{~nm}$

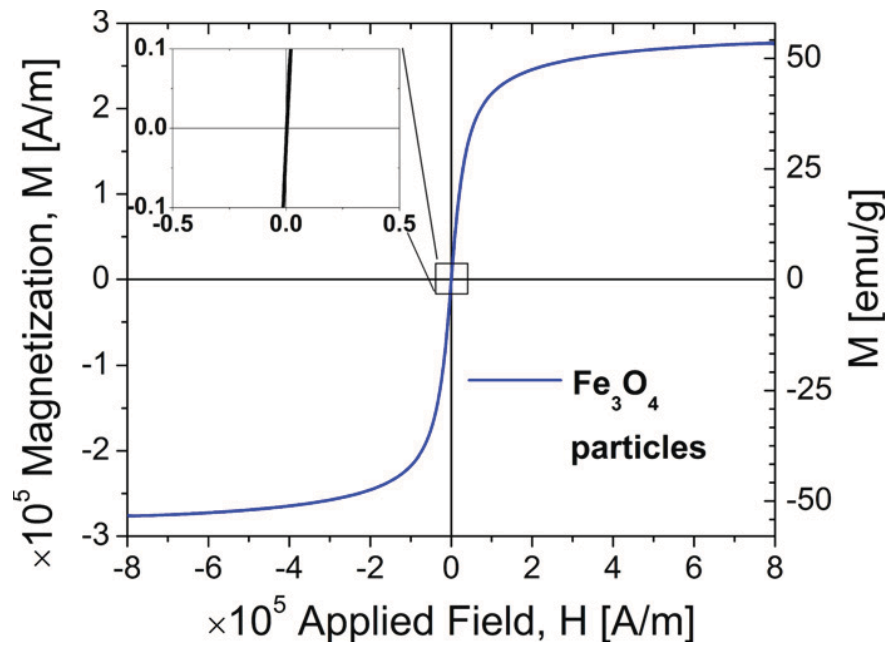

Fig. 10. Magnetization of $\mathrm{Fe}_{3} \mathrm{O}_{4}$ nanoparticles measured by vibrating sample magnetometer at room temperature. The particles show superparamagnetic behavior.

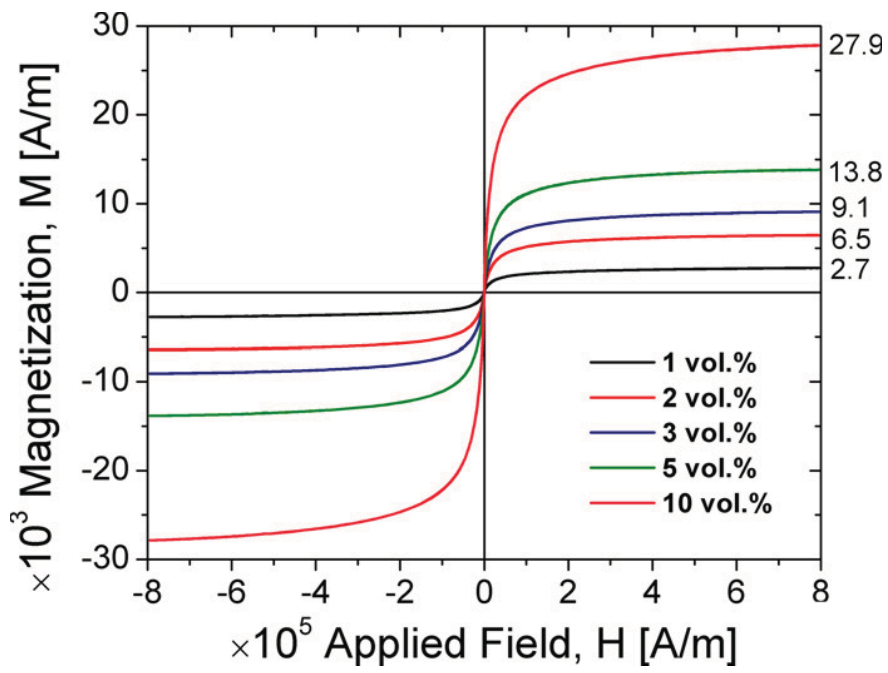

Fig. 11. Magnetization measurements of the composite with different $\mathrm{Fe}_{3} \mathrm{O}_{4}$ particles concentration at room temperature. All composites have no remanent magnetization and are superparamagnetic.

magnetite particles) [30]. The saturation magnetization of the particles depends on the particle size and the spin surface disorders. A presence of maghemite can lower magnetization. Maghemite nanoparticles produced with flame aerosol technology with similar diameters $(14 \pm 0.8 \mathrm{~nm})$ show a magnetization of $34 \mathrm{emu} \mathrm{g}^{-1}$ [31]. In Fig. 11, the M-H curves for the composite films with different nanoparticle concentrations are shown. All of them show superparamagnetic behavior at room temperature. When the magnetization values of the different composites are scaled to $100 \%$, the average saturation magnetization is found as $291 \mathrm{kA} \mathrm{m}^{-1}$ and is in agreement with the value of saturation magnetization of the initial particles.

\subsection{Influence of particle concentration on microstructure fabrication}

The SU-8 based nanocomposite is spin-coated and structured by conventional photolithography (exposure through a mask with a UV lamp). However, there are limitations of the particle concentration in the composite caused by UV-light absorption of the magnetite particles. The dependence of the transmittance on the composites' thickness is presented elsewhere [23]. Here, the 


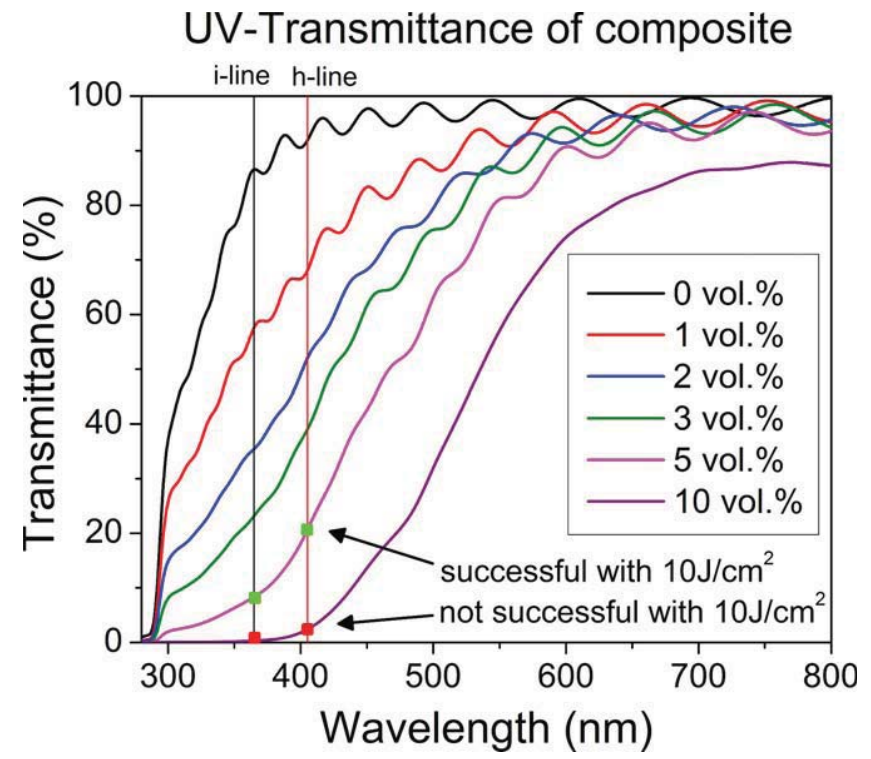

Fig. 12. UV-transmittance measurements of composite with increasing $\mathrm{Fe}_{3} \mathrm{O}_{4}$ particle concentration. The sinusoidal distortion of the signal is based on a Fabry-Perot effect which occurs when the thickness of the film is a multiple of the half wavelength. The thickness and the exposure dose of the samples are listed in Table 2.

UV/VIS-transmittance of the composite with different particle concentration was investigated (Fig. 12). The thickness of the samples considered was $1.65 \pm 0.15 \mu \mathrm{m}$, except for the $10 \mathrm{vol} \%$ case which was $2.2 \mu \mathrm{m}$ (Table 2). Higher nanoparticle content leads to a higher absorption in the UV region. For the composite exposure, a mercury lamp is used with two main spectral lines: i-line $365 \mathrm{~nm}$ and $\mathrm{h}$ line $405 \mathrm{~nm}$. Magnetite particles in the composite absorb in this
Table 2

Fabrication parameters of composite with different particle concentrations. The viscosity is measured at $5 \mathrm{kHz}$. Due to the increase of viscosity with the particle concentration the spin speed has to be increased to keep layer thicknesses in a similar range.

\begin{tabular}{lllcl}
\hline $\begin{array}{l}\text { Concentration } \\
{[\text { vol.\%] }}\end{array}$ & $\begin{array}{l}\text { Dynamic } \\
\text { viscosity at } \\
22.5^{\circ} \mathrm{C}[\mathrm{Pa} \text { s] }\end{array}$ & $\begin{array}{l}\text { Spin speed } \\
{[\mathrm{rpm}]}\end{array}$ & $\begin{array}{l}\text { Exposure dose } \\
{\left[\mathrm{mJ} \mathrm{cm}^{-2}\right]}\end{array}$ & $\begin{array}{l}\text { Fabricated } \\
\text { layer thickness } \\
{[\mu \mathrm{m}]}\end{array}$ \\
\hline 0 & 0.062 & 4000 & 200 & $1.54 \pm 0.01$ \\
1 & 0.065 & 4200 & 2000 & $1.64 \pm 0.01$ \\
2 & 0.065 & 4400 & 2000 & $1.57 \pm 0.02$ \\
3 & 0.078 & 4600 & 5000 & $1.61 \pm 0.01$ \\
5 & 0.106 & 5000 & 10,000 & $1.79^{\mathrm{c}} \pm 0.02$ \\
10 & n.a. $^{\text {a }}$ & 5000 & $10,000^{\mathrm{b}}$ & $2.20^{\mathrm{c}} \pm 0.03$ \\
\hline
\end{tabular}

a Data not available.

b Not fully polymerized.

Bigger thickness because of spin speed limitation.

area and can hinder polymerization, creating a limit in particle concentration. For a given layer thickness exposure doses for full polymerization must be increased with increasing particle concentration (Table 2).

Fig. 13 shows secondary electron microscope (SEM) images of the tip of fabricated magnetic composite cantilevers. Fig. 13(a) shows the top and Fig. 13(b) the bottom side of a fabricated cantilever tip containing 5 vol.\% magnetite particles, an exposure dose $D$ of $10 \mathrm{~J} \mathrm{~cm}^{-2}$, and a thickness of $1.8 \mu \mathrm{m}$ [23]. The UV transmittance of the composite layer is $8 \%$ at $365 \mathrm{~nm}$ and $21 \%$ at $405 \mathrm{~nm}$ (Fig. 12). Changing the particle concentration of the composite to $10 \mathrm{vol} . \%$ leads to a smooth top layer but to a rough and porous bottom layer (Fig. 13(c) and (d)). The rough bottom layer is caused by insufficient exposure dose because of UV absorption by the filler particles (transmittance of the composite layer is $0.3 \%$ at $365 \mathrm{~nm}$ and $2.4 \%$ at $405 \mathrm{~nm}$ ). A partially exposed bottom layer results in peeling of the
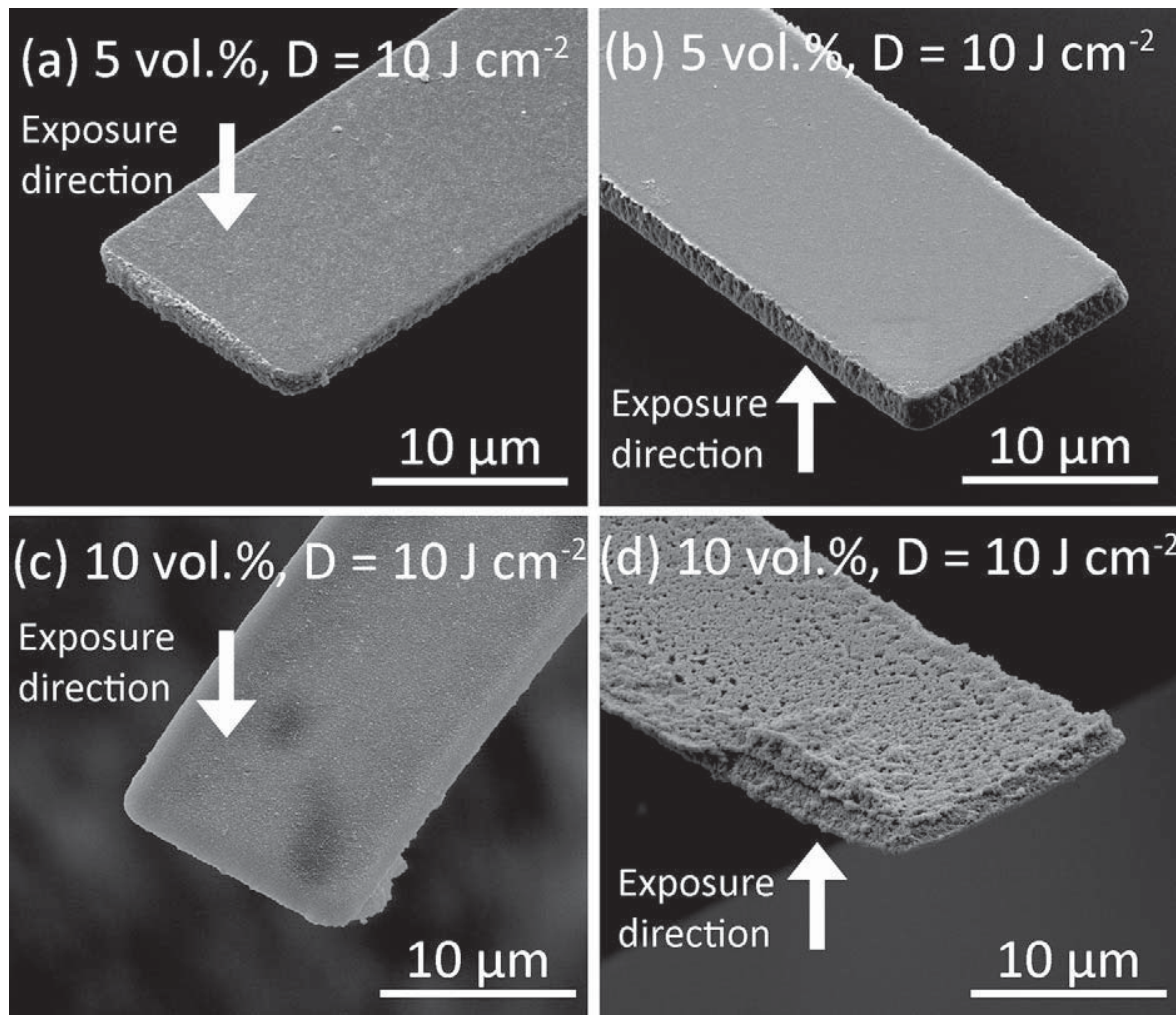

Fig. 13. SEM images of a composite microcantilever tip exposed with a dose of $10 \mathrm{~J} \mathrm{~cm}^{-2}$. Image (a) shows the top surface and (b) the bottom surface of a tip with 5 vol.\% $\mathrm{Fe}_{3} \mathrm{O}_{4}$ particle content. Both surfaces are smooth and fully exposed. A cantilever tip with a concentration of $10 \mathrm{vol}^{\circ} \% \mathrm{Fe}_{3} \mathrm{O}_{4}$ is shown in (c) presenting the top surface and in (d) the bottom surface. Due to the high absorption of the $\mathrm{Fe}_{3} \mathrm{O}_{4}$ particles the lower part of the composite cantilever is partially exposed and results in a rough bottom surface. 


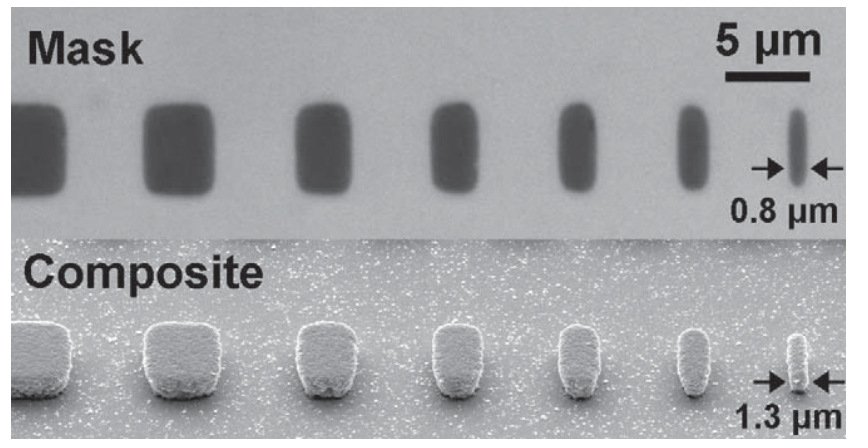

Fig. 14. The upper picture shows an optical microscope image of the resolution test pattern on the mask with different slit openings. On the lower picture a SEM view of the fabricated composite ( $5 \mathrm{vol} . \%$ ) pattern is shown. To see the side wall of the composite structures the fabricated composite sample is tilted by $45^{\circ}$. The narrowest pattern on the mask is $0.8 \mu \mathrm{m}$ and is successful transferred to the composite with an increased width of $1.3 \mu \mathrm{m}$ (due to dispersion effects during exposure).

structure during the baking process. An exposure dose of $10 \mathrm{~J} \mathrm{~cm}^{-2}$ corresponds to an approximately $20 \mathrm{~min}$ exposure time. Also, with increasing the exposure dose to $20 \mathrm{~J} \mathrm{~cm}^{-2}$ it was not possible to ensure a full polymerization of the cantilevers.

Additionally, the particle concentration influences the viscosity of the mixed composite. Viscosity is a crucial parameter for spincoating which determines the final layer thickness. Higher particle concentrations increase the viscosity as seen in Table 2 . The spin speed must be adjusted to fabricate composite layers with similar layer thicknesses. For particle concentrations of $5 \mathrm{vol} . \%$ and $10 \mathrm{vol} . \%$, the maximum spin speed of $5000 \mathrm{rpm}$ of the spin coater was reached and the target thickness of $1.6 \mu \mathrm{m}$ could no longer be adjusted.

\subsection{Pattern transfer in composite microstructures}

To determine the resolution of the composite, test structures with different patterns on the mask have been designed. Fig. 14 shows the mask pattern and the resulting composite structures ( 5 vol.\%, thickness: $1.8 \mu \mathrm{m}$, spinspeed: $5000 \mathrm{rpm}$, exposure dose: $10 \mathrm{~J} \mathrm{~cm}^{-2}$ ). The smallest structure size on the mask, having a width of $0.8 \pm 0.1 \mu \mathrm{m}$, is successfully transferred into the composite with a width of $1.3 \pm 0.2 \mu \mathrm{m}$. This shows that the resolution limit of the composite was not reached. The fabricated composite structures are in general $0.6 \pm 0.2 \mu \mathrm{m}$ wider, most likely resulting from the light dispersion in the composite by the incorporated nanoparticles. The slight v-shape of the composite structures can result from the absorption of UV light in the upper part of the composite layer by the nanoparticles. It is known that bare SU-8 structures can exhibit a negative slope as well because of the absorption of deep UV light in the upper layer. Using a UV-filter to remove the sub-365 nm light could help reduce the effect of the negative slope [32,33].

\subsection{Surface properties}

With dynamic water contact angle measurements on samples with $0,1,2,3,5,10$ vol.\%, no influence of the particle concentration on surface chemistry and surface roughness on the surface of the composite could be detected [34]. The advancing contact angle which indicates the hydrophobicity of the sample is $81.1 \pm 1.6^{\circ}$ for all samples and does not differ significantly from the unfilled samples $81.8 \pm 1.3^{\circ}$. If the particle concentration would increase surface roughness, then the contact angle hysteresis (advancing minus receding contact angle) would also increase. However, the hysteresis was for all samples between $29^{\circ}$ and $40^{\circ}$, and no distinct dependence on the particle concentration could be detected.

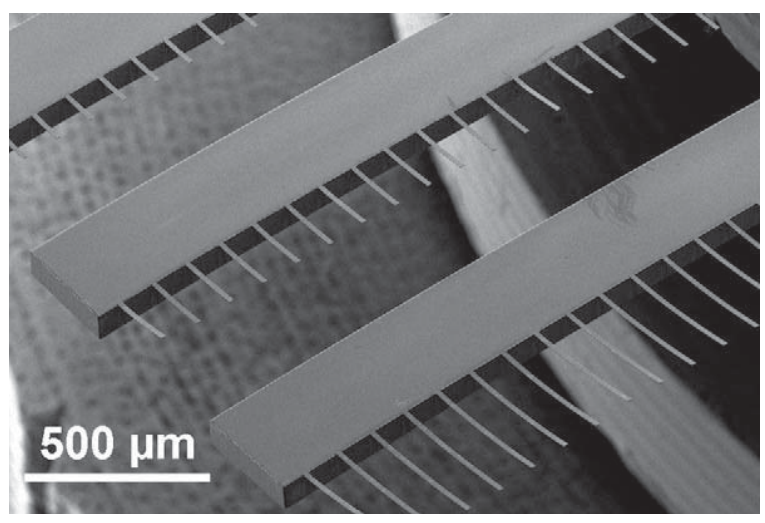

Fig. 15. SEM image of fabricated cantilever array with magnetic polymer composite filled with 5 vol.\% of magnetite nanoparticles.

\subsection{Young's modulus}

Microcantilever arrays with different particle concentration have been successful fabricated (Fig. 15). The dynamic Young's modulus $\mathrm{E}$ of the composites with different particle concentration can be determined by measuring the resonant frequency of the fabricated composite cantilevers and the Euler Bernoulli beam equation

$E=12 \rho\left(\frac{2 \pi L^{2} f_{1}}{\lambda_{1}^{2} h}\right)^{2}$,

where $L$ is the length of the cantilever, $f_{1}$ the first resonant frequency, $h$ the thickness of the cantilever, and $\lambda_{1}=1.875$ the eigenvalue of the first resonant frequency. The cantilevers were actuated by thermal noise in vacuum to avoid damping by the environment and the resonance frequency is measured by a laserDoppler interferometer. The measured Young's moduli of different particle concentration are plotted in Fig. 16. The cantilevers with nanoparticle concentrations varying from 0 to 3 vol.\% show the same characteristics and have a mean Young's modulus of $4.4 \mathrm{GPa}$. The cantilevers with a fill of 5 vol.\% show a slightly higher mean

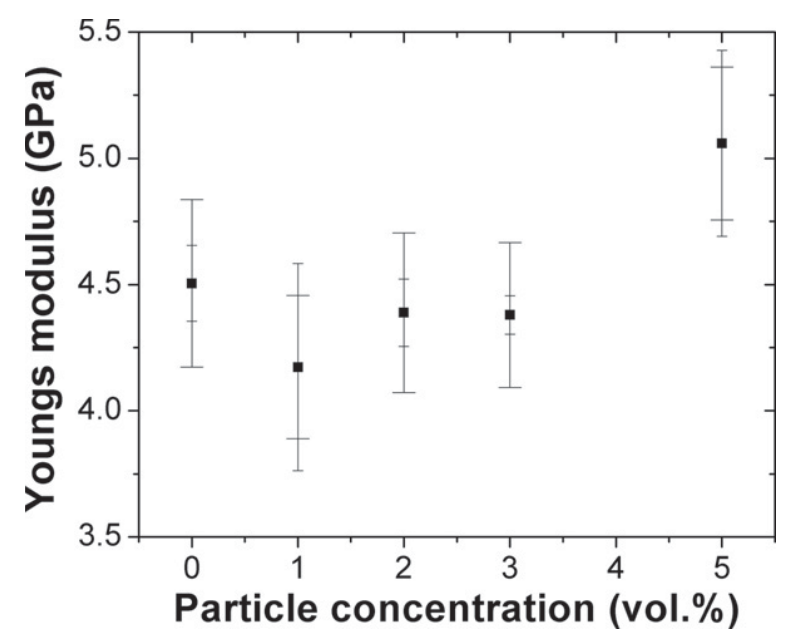

Fig. 16. Dynamic Young's Modulus measurements of composite cantilevers with different particle concentration $0,1,2,3,5$ vol.\% dependent on the frequency (lengths) of the cantilevers. The Young's modulus is extracted from resonant frequency measurement of the cantilevers by a laser-Doppler vibrometer and using Euler Bernoulli beam theory. For each fill grade 9-25 cantilevers with the same length from the same device and batch, respectively, are measured (see also Fig. 15). All cantilevers have frequencies between 23 and $47 \mathrm{kHz}$ (lengths: $100-160 \mu \mathrm{m}$ ). The standard deviations of the measurements are indicated with the small error bars and the calculated "measurement error" with the large error bars. 
Young's modulus of 5.1 GPa. The standard deviations of cantilevers with the same length and same nanoparticle fill are between 0.1 and $0.4 \mathrm{GPa}$, and the corresponding calculated measurement errors are smaller than $0.4 \mathrm{GPa}$. The measured dynamic Young's modulus of bare SU-8 is in good agreement with the measurements of the dynamic Young's modulus ( $4.5 \mathrm{GPa}$ at $25 \mathrm{kHz}$ ) using cantilevers actuated by the Kelvin polarization force [35]. Comparison of the measured Young's modulus of filled polymer with the literature is difficult as it is known that particle morphology, surfactant and different baking time and temperature for photocurable polymer affect mechanical reinforcing. It is reported that adding silica nanoparticles in photocurable epoxy slightly increases the static Young's modulus (measured by nanoindentation) [6], while there is a significant increase in Young's modulus with silica in PDMS [36].

\section{Conclusion}

A photopatternable superparamagnetic nanocomposite for the fabrication of microstructures has been developed and characterized. A high magnetic particle concentration in the composite is important to obtain high magnetic forces on the microstructures. The influence of the magnetite nanoparticle concentration on the dispersion as well as on the fabrication steps were investigated and optimized for homogeneity and structural integrity. With the use of a phosphate-based polymeric dispersing agent, nanocomposite films with particle concentration up to 10 vol.\% (32 wt.\%) and a low amount of agglomerates with mean sizes around $50 \mathrm{~nm}$ were obtained. SAXS measurements show that agglomerates are independent of the amount of embedded particles. XDC measurements indicate that the agglomerates were already present in the initial magnetic suspension and do not agglomerate significantly during the mixing of the composite. The UV exposure dose was optimized to fabricate cantilevers by conventional photolithography with different particle concentrations. Microstructures with up to 5 vol.\% of magnetite compromise the magnetic properties with the full polymerization of the microstructures. A saturation magnetization of $13.8 \mathrm{kA} \mathrm{m}^{-1}$ was measured for the composite film with $5 \mathrm{vol} . \%$ particles. Microstructures with widths down to $1.3 \mu \mathrm{m}$ (thickness of $1.8 \mu \mathrm{m}$ ) could be fabricated with 5 vol.\% $\mathrm{Fe}_{3} \mathrm{O}_{4}$ nanoparticles without reaching the resolution limit of the composite. With the succesfull fabricated cantilever arrays the composite's dynamic Young's modulus was determined. A slight increase in the Young's modulus ( $5.1 \mathrm{GPa}$ ) was observed for composites containing 5 vol.\% particles.

Using a remote magnetic actuation and an optical read out to detect a resonance frequency shift, such microcantilevers can be used as mass sensors or micro balances. With an appropriate functionalization of the cantilever polymer surface with target receptors, these cantilevers are predestined to study biomolecules for example to investigate antibody-antigen interactions.

\section{Acknowledgements}

The authors acknowledge Eszter Barthazy and Elisabeth Müller (EMEZ, ETH) for the TEM-images, Doris Spori (Surface Science \& Technology, ETH) for the contact angle measurements and Prof. Ann Marie Hirt (EPM, ETH) for the support with the magnetic measurements. The authors would also like to thank Christian Bergemann from Chemicell $\mathrm{GmbH}$ for the cooperation preparing the magnetic particle dispersion. Furthermore, the authors thank Michael Wendlandt for his fundamental inputs and Eva Pellicer from the Laboratori de Mesures Tèrmiques i Magnètiques (Autonomous University of Barcelona) for her fruitful comments on the analysis of the composites. Financial support by the ETH Zurich (TH-28 06-3) and SNSF (Project No. 200020-113350) are acknowledged.

\section{Appendix A. Supplementary data}

Supplementary data associated with this article can be found, in the online version, at doi:10.1016/j.snb.2011.04.078.

\section{References}

[1] R. Bashir, BioMEMS: state-of-the-art in detection, opportunities and prospects, Advanced Drug Delivery Reviews 56 (2004) 1565-1586.

[2] S. Ramakrishna, J. Mayer, E. Wintermantel, K.W. Leong, Biomedical applications of polymer-composite materials: a review, Composites Science and Technology 61 (2001) 1189-1224.

[3] R.T. Olsson, M.A.S. Azizi Samir, G. Salazar Alvarez, L. Belova, V. Strom, L.A. Berglund, O. Ikkala, J. Nogues, U.W. Gedde, Making flexible magnetic aerogels and stiff magnetic nanopaper using cellulose nanofibrils as templates, Nature Nano 5 (2010) 584-588.

[4] D. Godovsky, Device applications of polymer-nanocomposites, in: Advances in Polymer Science, Springer, Berlin/Heidelberg, 2000, pp. 163-205.

[5] S. Jiguet, A. Bertsch, M. Judelewicz, H. Hofmann, P. Renaud, SU-8 nanocomposite photoresist with low stress properties for microfabrication applications, Microelectronic Engineering 83 (2006) 1966-1970.

[6] A. Voigt, M. Heinrich, C. Martin, A. Llobera, G. Gruetzner, F. Pérez-Murano, Improved properties of epoxy nanocomposites for specific applications in the field of MEMS/NEMS, Microelectronic Engineering 84 (2007) 10751079 .

[7] S. Jiguet, A. Bertsch, H. Hofmann, P. Renaud, Conductive SU8 photoresist for microfabrication, Advanced Functional Materials 15 (2005) 1511-1516.

[8] M. Feldmann, S. Buttgenbach, Novel microrobots and micromotors using Lorentz force driven linear microactuators based on polymer magnets, IEEE Transactions on Magnetics 43 (2007) 3891-3895.

[9] N. Damean, B.A. Parviz, J.N. Lee, T. Odom, G.M. Whitesides, Composite ferromagnetic photoresist for the fabrication of microelectromechanical systems, Journal of Micromechanics and Microengineering 15 (2005) 29-34.

[10] T. Phenrat, H.J. Kim, F. Fagerlund, T. Illangasekare, R.D. Tilton, G.V. Lowry, Particle size distribution, concentration, and magnetic attraction affect transport of polymer-modified Fe-0 nanoparticles in sand columns, Environmental Science \& Technology 43 (2009) 5079-5085.

[11] Q.Y.Zhang, H.P.Zhang, G. Xie, J.P. Zhang, Effect of surface treatment of magnetic particles on the preparation of magnetic polymer microspheres by miniemulsion polymerization, Journal of Magnetism and Magnetic Materials 311 (2007) 140-144.

[12] A.H. Lu, E.L. Salabas, F. Schuth, Magnetic nanoparticles: synthesis, protection, functionalization, and application, Angewandte Chemie-International Edition 46 (2007) 1222-1244.

[13] P.C. Gach, C.E. Sims, N.L. Allbritton, Transparent magnetic photoresists for bioanalytical applications, Biomaterials 31 (2010) 8810-8817.

[14] A. del Campo, C. Greiner, SU-8: a photoresist for high-aspect-ratio and 3D submicron lithography, Journal of Micromechanics and Microengineering 17 (2007) R81-R95.

[15] G. Kotzar, M. Freas, P. Abel, A. Fleischman, S. Roy, C. Zorman, J.M. Moran, J. Melzak, Evaluation of MEMS materials of construction for implantable medical devices, Biomaterials 23 (2002) 2737-2750.

[16] M. Hennemeyer, F. Walther, S. Kerstan, K. Schürzinger, A.M. Gigler, R.W. Stark, Cell proliferation assays on plasma activated SU-8, Microelectronic Engineering 85 (2008) 1298-1301.

[17] Z.-Z. Wu, Y. Zhao, W.S. Kisaalita, Interfacing SH-SY5Y human neuroblastoma cells with SU-8 microstructures, Colloids and Surfaces B: Biointerfaces 52 (2006) 14-21.

[18] S. Schmid, P. Wagli, C. Hierold, Biosensor based on all-polymer resonant microbeams, in: IEEE 22nd Int. Conf. on Micro Electro Mechanical Systems, 2009, pp. 300-303.

[19] W.H. Teh, U. Durig, U. Drechsler, C.G. Smith, H.J. Guntherodt, Effect of low numerical-aperture femtosecond two-photon absorption on (SU-8) resist for ultrahigh-aspect-ratio microstereolithography, Journal of Applied Physics 97 (2005) 054907

[20] D.R. Lide, Handbook of Chemistry and Physics, 78 ed., CRC Press, Boca Raton FL 1997.

[21] H.C. Chiamori, J.W. Brown, E.V. Adhiprakasha, E.T. Hantsoo, J.B. Straalsund, N.A Melosh, B.L. Pruitt, Suspension of nanoparticles in SU-8: processing and characterization of nanocomposite polymers, Microelectronics Journal 39 (2008) 228-236.

[22] S. Jiguet, A. Bertsch, H. Hofmann, P. Renaud, SU8-silver photosensitive nanocomposite, Advanced Engineering Materials 6 (2004) 719-724.

[23] M. Suter, O. Ergeneman, J. Zürcher, S. Schmid, A. Camenzind, B.J. Nelson, C. Hierold, Superparamagnetic photocurable nanocomposite for the fabrication of microcantilevers, Journal of Micromechanics and Microengineering 21 (2011) 025023.

[24] R.W. Cheary, A.A. Coelho, An experimental investigation of the effects of axial divergence on diffraction line profiles, Powder Diffraction 13 (1998) 100-106.

[25] TJ. Daou, S. Begin-Colin, J.M. Grenèche, F. Thomas, A. Derory, P. Bernhardt, P. Legaré, G. Pourroy, Phosphate adsorption properties of magnetite-based nanoparticles, Chemistry of Materials 19 (2007) 4494-4505.

[26] W.B. Russel, D.A. Saville, W.R. Schowalter, Colloidal Dispersions, Cambridge University Press, Cambridge, 1989. 
[27] O. Glatter, A new method for the evaluation of small-angle scattering data, Journal of Applied Crystallography 10 (1977) 415-421.

[28] O. Glatter, Determination of particle-size distribution functions from smallangle scattering data by means of the indirect transformation method, Journal of Applied Crystallography 13 (1980) 7-11.

[29] H. Borchert, E.V. Shevchenko, A. Robert, I. Mekis, A. Kornowski, G. Grübel, H. Weller, Determination of nanocrystal sizes: a comparison of TEM, SAXS, and XRD studies of highly monodisperse $\mathrm{CoPt}_{3}$ particles, Langmuir 21 (2005) 1931-1936.

[30] G.F. Goya, T.S. Berquo, F.C. Fonseca, M.P. Morales, Static and dynamic magnetic properties of spherical magnetite nanoparticles, Journal of Applied Physics 94 (2003) 3520-3528.

[31] A. Teleki, M. Suter, P.R. Kidambi, O. Ergeneman, F. Krumeich, B.J. Nelson, S.E. Pratsinis, Hermetically coated superparamagnetic $\mathrm{Fe}_{2} \mathrm{O}_{3}$ particles with $\mathrm{SiO}_{2}$ nanofilms, Chemistry of Materials 21 (2009) 2094-2100.

[32] N.E. Antolino, G. Hayes, R. Kirkpatrick, C.L. Muhlstein, M.I. Frecker, E.M. Mockensturm, J.H. Adair, Lost mold rapid infiltration forming of mesoscale ceramics. Part 1. Fabrication, Journal of the American Ceramic Society 92 (2009) S70-S78.

[33] D.M. Spori, T. Drobek, S. Zürcher, M. Ochsner, C. Sprecher, A. Mühlebach, N.D. Spencer, Beyond the lotus effect: roughness influences on wetting over a wide surface-energy range, Langmuir 24 (2008) 5411-5417.

[34] D.M. Spori, T. Drobek, S. Zurcher, N.D. Spencer, Cassie-state wetting investigated by means of a hole-to-pillar density gradient, Langmuir 26 (2010) 9465-9473.

[35] S. Schmid, A. Leimbacher, C. Hierold, Integrated dynamic mechanical testing of polymer micro structures, in: Euspen International Conference, Zurich, 2008, pp. 447-473.

[36] A. Camenzind, T. Schweizer, M. Sztucki, S.E. Pratsinis, Structure and strength of silica-PDMS nanocomposites, Polymer 51 (2010) 1796-1804.

\section{Biographies}

Marcel Suter received his Dipl. Ing. FH in System Engineering at the Interstate University of Applied Sciences of Technology Buchs NTB. Then he completed a B.Sc. and M.Sc. at the University of Neuchâtel in Micro- and Nanotechnology. He wrote his diploma thesis at University of California Berkeley BSAC (USA). Since 2007 he is a PhD student in the Micro- and Nanosystems Group at ETH Zurich, working on magnetic polymer actuators.

OlgaçErgeneman received the B.S. degree from the Department of Electrical and Electronics Engineering, Middle East Technical University, Ankara, Turkey, in 2003, and the M.S. degree from the Department of Electrical Engineering, Koç University, Istanbul, Turkey, focusing on design and fabrication of polymer magnetic actuators for scanning applications. Since 2005, he has been working toward the Ph.D. degree in the Institute of Robotics and Intelligent Systems, ETH, Zurich, Switzerland. His research interests are MEMS, magnetic actuators, microfabrication, electroplating, and optical sensors.

Jonas Zürcher received the B.Sc. degree in mechanical and process engineering from the Swiss Federal Institute of Technology (ETH), Zürich, Switzerland, in 2009. He is currently working toward the M.Sc degree in micro- and nanosystems from the Swiss Federal Institute of Technology (ETH), Zürich, Switzerland.

Christian Moitzi received a Ph.D. in Physical Chemistry from the University of Graz in Austria. He was working at the Soft Condensed Matter group at the Department of Physics of the University of Fribourg in Switzerland. He also served as laboratory head at the Soft Nanoscience group of the AdolpheMerkle Institute of the University of Fribourg. Currently, he is Product Specialist for Small Angle X-Ray Scattering at Anton Paar $\mathrm{GmbH}$.

Salvador Pané received the B.S. and M.S. degrees in chemistry and the Ph.D. degree, working on alloys and magnetic composites obtained by electrochemical deposition, from the University of Barcelona, Barcelona, Spain, in 2003, 2004, and 2007, respectively. Since May 2007, he has been with the Institute of Robotics and Intelligent Systems, ETH, Zurich, Switzerland, as a Postdoctoral Researcher. His research interests are micro- and nanorobotics with a focus on magnetic micro- and nanostructures and coatings for targeted drug delivery.

Thomas Rudin got his diploma in Mechanical \& Process Engineering from the Swiss Federal Institute of Technology ETH Zurich in 2006. Since 2007 he is a PhD student in the Particle Technology Laboratory at ETH Zurich, working on flame synthesis of nanoparticles.

Sotiris E. Pratsinis (Ph.D. UCLA, 1985) was professor (1985-2000) at the University of Cincinnati and since 1998 Professor of Mechanical \& Process Engineering and Adjunct Professor of Materials Science at ETH Zurich, Switzerland. He has graduated $24 \mathrm{Ph} . \mathrm{D}$. students and currently advises 11 . He has published over 300 articles and been awarded 20 patents licensed to industry that have contributed also to formation of four spinoffs. He is on the Editorial Board of six journals and has received a 1989 Presidential Young Investigator Award by the U.S. NSF and a 2009 Advanced Investigator Grant by the European Research Council.

Brad Nelson received mechanical engineering degrees from the University of Illinois (B.S. 1984) and the University of Minnesota (M.S. 1987), and a Ph.D. in Robotics (School of Computer Science) from Carnegie Mellon University (1995). Prof. Nelson has been on the faculty of the University of Minnesota and the University of Illinois at Chicago, has worked at Motorola and Honeywell, and has served as a United States Peace Corps Volunteer in Botswana, Africa. He is the Professor of Robotics and Intelligent Systems at the Swiss Federal Institute of Technology (ETH), Zurich and heads the Institute of Robotics and Intelligent Systems (IRIS).

Christofer Hierold is Professor of Micro- and Nanosystems at ETH Zurich. His major research at ETH Zurich is focused on the field of nanotransducers, new materials for MEMS and advanced microsystems. He has been serving in program committees of numerous scientific conferences; he is co-chair of the Steering Committee of the European Conference on Solid-State Transducers (Eurosensors) and he is member of the Steering Committee of the International Conference on Solid-State Sensors, Actuators and Microsystems (Transducers) and of the International Conference on Micro Electro Mechanical Systems (MEMS). He is member of the editorial boards of the IEEE/ASME Journal of Micro Electromechanical Systems and of the IoP Journal of Micromechanics and Microengineering, and he is joint editor of Wiley-VCH's book series on Advanced Micro and Nanosystems. Christofer Hierold is member of the Swiss Academy of Engineering Sciences (SATW). 